\title{
hsa-miR-195-5p inhibits cell proliferation of human thyroid carcinoma cells via modulation of p21/cyclin D1 axis
}

\author{
Dexin Liu, Ping Li, Xiaodong Wang, Wei Wang \\ Department of Radiology, Second Affiliated Hospital of Fujian Medical University, Quanzhou, China \\ Contributions: (I) Conception and design: D Liu, W Wang; (II) Administrative support: D Liu; (III) Provision of study materials: W Wang; (IV) \\ Collection and assembly of data: P Li; (V) Data analysis and interpretation: X Wang; (VI) Manuscript writing: All authors; (VII) Final approval of \\ manuscript: All authors. \\ Correspondence to: Wei Wang. Department of Hepatobiliary Pancreatic Surgery, Second Affiliated Hospital of Fujian Medical University, 34 N. \\ Zhongshan Road, Quanzhou, China. Email: gianty2005@yahoo.com.
}

Background: Based on existing evidence, microRNAs (miRs) are gene regulators that undertake key functions in the oncogenesis and tumor progression of every single human malignant disease, such as thyroid carcinoma (TC). Previous clinical findings showed that expression of miR-195 is down-regulated in TC, which implies that miR-195 may be practically involved in TC pathogenesis. Nevertheless, the function of hsa-miR-195-5p in TC is still largely unclear. Herein, we detected the conceivable involvement of hsa-miR$195-5 \mathrm{p}$ in TC cell proliferation.

Methods: Real time PCR examination was performed to assess the expression level of hsa-miR-195-5p in TC cell lines TPC-1 and B-CPAP. TPC-1 cells were transfected with either hsa-miR-195-5p mimics or hsa-miR-195-5p inhibitor. After confirmation of transfection efficiency, the effect of hsa-miR-195$5 \mathrm{p}$ on proliferation and cell cycle of TPC-1 cells was assessed. The expression of cyclin D1 and p21 was simultaneously detected by western blotting. Moreover, targetScan 6.2 was used to predict hsa-miR-195-5p target genes. Subsequently, luciferase reporter was performed to examine whether there is a possible binding of hsa-miR-195-5p to 3'-UTR of cyclin D1 mRNA. Furthermore, cyclin D1 mRNA and protein levels were measured to check whether hsa-miR-195-5p exerts its function at the post-transcriptional level. In addition, to explore the function of cyclin D1 in TPC-1 cells overexpressing hsa-miR-195-5p, cyclin D1 siRNA was used to silence the expression of cyclin D1 in TPC-1 cells overexpressing hsa-miR-195-5p.

Results: We quantified the expression of hsa-miR-195-5p in TC cells and normal thyroid cells and found a remarkable decrease in hsa-miR-195-5p expression in TC cells. Over-expression of hsa-miR-195-5p obviously resulted in downgraded proliferation of TC cells. Moreover, hsa-miR-195-5p caused cell arrest at the GO/G1 phase. Further in silico analyses and the dual-luciferase reporter assay confirmed that 3'-UTR of cyclin D1 is a direct target of hsa-miR-195-5p. Western blot analysis uncovered that hsa-miR-195-5p overexpression led to decreased levels of cyclin D1 and p21. In mechanistic analyses, we found that silencing of cyclin D1 reversed the inhibitory effect of hsa-miR-195-5p on the proliferation of TC cells, which indicates that hsa-miR-195-5p suppresses TC cell proliferation by adversely regulating cyclin D1.

Conclusions: We concluded that hsa-miR-195-5p is a candidate tumor-suppressor miRNA in TC and that the hsa-miR-195-5p/p21/cyclin D1 pathway could be a potential therapeutic target for TC.

Keywords: Cyclin D1; cell cycle; cell proliferation; hsa-miR-195-5p; thyroid carcinoma (TC)

Submitted Feb 19, 2020. Accepted for publication Jul 17, 2020.

doi: $10.21037 /$ tcr-20-1083

View this article at: http://dx.doi.org/10.21037/tcr-20-1083 


\section{Introduction}

Thyroid cancer (TC), one of the sporadic human carcinomas and representing about $5 \%$ of thyroid nodules, is the most widespread endocrine cancer around the world (1). The incidence rate of TC in developed countries and developing countries such as China has been undergoing a marked global upsurge in recent decades (2-5), making TC an emerging public health concern. Recent advances in TC treatment such as chemotherapy or surgery have improved the prognosis of TC (6). Notwithstanding, due to the lack of indepth understanding of the molecular mechanism on the occurrence and development of the disease, patients with advanced TC still have few treatment alternatives. Therefore, it is of far-reaching clinical significance to explore novel molecular targets for TC therapy.

A growing body of evidence suggested that microRNAs (miRNAs) are able to control the expression of genes related to proliferation, invasion and migration of cancer cells by targeting mRNA 3'UTRs, so as to prevent or accelerate the progress of tumor (7-15). Previous medical and basic research data suggested that miR-195 plays an important role in cancer (16-26). The expression of miR-195-5p was previously reported to be elevated in several diseases, such as deep vein thrombosis (27), while in other diseases, including collapse of steroid-induced osteonecrosis of the femoral head, non-small cell lung cancer and cervical carcinoma, decreased expression of miR-195-5p was confirmed (28-30). In particular, it has been recently reported that miR-195 expression in TC tissue is decreased, and its ectopic high expression has been proved to significantly inhibit the expression of raf1, thus inhibiting the proliferation of TC cells (31). More recent studies showed that miR-195 acts as a tumor suppressor in TC by inhibiting tumor growth and metastasis, which is probably related to regulation of $\mathrm{Wnt} / \beta$-catenin signaling pathway, VEGF-A and p53 in mechanism $(32,33)$. Moreover, when the function of miR195 is interfered by other factors, the malignancy of TC cells is remarkably enhanced (34). All things considered, the link between TC and the expression of hsa-miR-195-5p is still largely obscure and poorly understood, especially in the aspect of what concerns its inhibitory effect on TC cell proliferation and the underlying molecular mechanism at cell cycle levels.

Along these lines, the aim of this study was to investigate the function of hsa-miR-195-5p in regulation of TC cell proliferation and the underlying molecular mechanism.

\section{Methods}

\section{Cell culture}

The human TC cell lines TPC-1 (RRID:CVCL_6298) as well as normal thyroid cell line HT-ori3 (RRID:CVCL_4W02) used in the present study were provided by American Type Culture Collection (Manassas, VA, USA). B-CPAP (DSMZ Cat\# ACC-273, RRID:CVCL_0153) were purchased from University of Colorado Cancer Center Cell Bank. Cells were cultured at $37{ }^{\circ} \mathrm{C}$ in a $5 \% \mathrm{CO}_{2}$ and $95 \%$ humidity incubator using DMEM medium (Gbico, USA) complemented with $10 \%$ FBS (Sigma, USA).

\section{Transient transfection}

hsa-miR-195-5p mimic, hsa-miR-195-5p inhibitor and the respective negative control oligonucleotides were purchased from GeneCopoeia (Guangzhou, China). The cyclin D1 siRNA (h): sc-29286 was obtained from Santa Cruz Biotechnology, Inc and used following the vendor's recommended protocol. Lipofectamine 2000 transfection reagent (Invitrogen, Carlsbad, CA, USA) was employed in line with the manufacturer's instructions.

\section{RNA extraction and real-time quantitative PCR}

The extraction of total RNA from cells was performed with mirVana miRNA Isolation Kit (Ambion) following the protocol described in the manufacturer provided manual. To determine the hsa-miR-195-5p expression levels, real time qRT-PCR experiments were carried out using the gene-specific TaqMan miRNA Assay Probes (Applied Biosystems, Foster City, CA, USA). The real-time PCR reaction was performed in triplicate. The relative hsa-miR195-5p expression was assessed by normalization to the small nuclear RNA U6 using the $2^{-\Delta \Delta \mathrm{Ct}}$ method.

\section{Western blotting}

Cells were lysed using a lysis buffer (Beyotime, Jiangsu, China) and proteins were separated by SDS-PAGE. Next, purified proteins were transferred onto nitrocellulose membranes followed by blocking in TBST containing 5\% non-fat milk for 2 hours. After an overnight incubation with primary antibodies against cyclin D1 (Abcam Cat\# AB 190194, RRID:AB_2728784), p21 (Abcam Cat\# ab188224, RRID:AB_2734729) or GAPDH (Abcam 


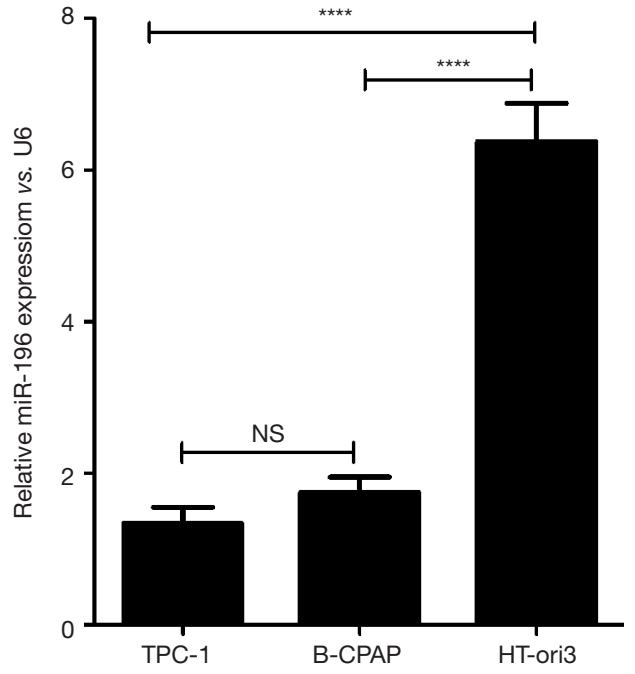

Figure 1 Expression of hsa-miR-195-5p in human thyroid carcinoma cell lines. Relative hsa-miR-195-5p mRNA expression levels in TC cell lines TPC-1 and B-CPAP and normal thyroid cell line HT-ori3 were determined by real time qRT-PCR. Experiments were repeated at least three times. Each bar represents the mean of three independent experiments. ${ }^{* * *}, \mathrm{P}<0.0001$; NS, non-significant in intergroup comparisons.

Cat\# ab9485, RRID:AB_307275), membranes were incubated with anti-rabbit HRP-conjugated secondary antibody (Millipore Cat\# 12-348, RRID:AB_390191). Next, membranes were washed with TBST and proteins were revealed by chemiluminesence. Image $\mathrm{J}$ was used for densitometry quantification analysis.

\section{MTT assay}

The MTT cell proliferation assay was performed using the TPC-1 cells. Briefly, TPC-1 cells (2,000 cells/well) were seeded into 96-well microplates in medium containing $10 \%$ FBS for 72 hours. Afterward, cells were stained with $100 \mu \mathrm{L}$ MTT reagent $(0.5 \mathrm{mg} / \mathrm{mL}$, Sigma, St Louis, MO, USA) and incubated for another $4 \mathrm{~h}$ at $37^{\circ} \mathrm{C}$. After discarding the culture medium and replacing $150 \mu \mathrm{L}$ DMSO as its substitution, the absorbance at $490 \mathrm{~nm}$ was assessed in a Thermo Scientific Multiskan (Thermo Fisher Scientific, USA).

\section{Luciferase assays}

Approximately $5 \times 10^{4}$ cells/well were cultured in 24-well microplates for 24 hours and then cells were co-transfected with hsa-miR-195-5p mimic or miR-NC and luciferase reporter vector containing the mutated cyclin D1 3'-UTR (cyclin D1-3'-UTR-mut) or the wild type cyclin D1 3'UTR (WT 3'-UTR) using Lipofectamine 2000 (Invitrogen, Carlsbad, CA, USA). The luciferase activity was measured after additional 48 hours incubation using the dual luciferase assay kit in line with the manufacturer's protocol (Promega, Wisconsin, WI, USA).

\section{Statistical analysis}

The statistical analysis was done using One-way or twoway ANOVA with bonferroni post test in GraphPad Prism Software for Windows, version 6.00, San Diego California USA (www.graphpad.com). $\mathrm{P}<0.05$ was the statistically significant cutoff $\mathrm{P}$ value.

\section{Results}

bsa-miR-195-5p expression was down-regulated in TC cell lines

The expression level of hsa-miR-195-5p in TC cell lines TPC- 1 and B-CPAP was assessed by real time PCR examination. The results (Figure 1) revealed that the relative expression of hsa-miR-195-5p was significantly down-regulated in both TPC-1 $(1.34 \pm 0.21)$ and B-CPAP $(1.75 \pm 0.20)$ cells compared with the normal thyroid cell line HT-ori3 (6.38 \pm 0.50$)$. These results reveal that hsa-miR$195-5 \mathrm{p}$ is abnormally down-regulated in human TC cell lines, indicating it could be involved in TC pathogenesis.

\section{bsa-miR-195-5p inbibited TC cell proliferation}

To evaluate whether hsa-miR-195-5p is involved in the proliferation phenotype of TC cells, TPC-1 cells were transfected with either hsa-miR-195-5p mimics or hsa-miR195-5p inhibitor. The relative expression of hsa-miR-195$5 \mathrm{p}$ in the transfected cells was detected by qRT-PCR. The results displayed that transfection of hsa-miR-195-5p mimic reestablished its expression, while hsa-miR-195-5p inhibitor efficiently suppressed this expression (Figure $2 A$ ). After having confirmed the transfection efficiency, we assessed the effects of hsa-miR-195-5p on the proliferation of TPC1 cells. Transfection with hsa-miR-195-5p mimics resulted in obvious inhibition of cell proliferation (Figure $2 B$ ). On the contrary, inhibition of hsa-miR-195-5p expression in TPC-1 cells could significantly increase cell proliferation 
A

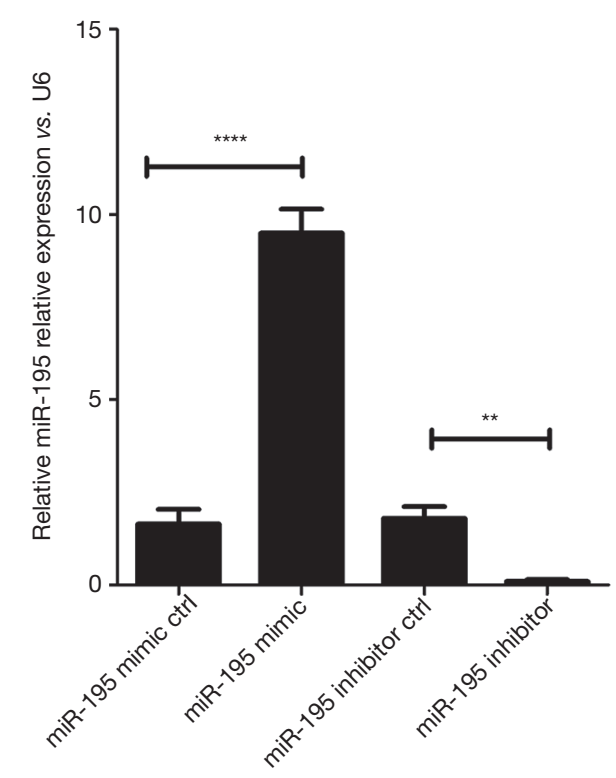

B



Figure 2 Exogenous over-expression of hsa-miR-195-5p inhibited TC cell proliferation. (A) Verification of hsa-miR-195-5p expression levels by PCR analysis after transfection. (B) MTT assays revealed that over-expression of hsa-miR-195-5p inhibited growth of TPC-1 cells while inhibition of hsa-miR-195-5p promoted growth of TPC-1 cells. Each bar represents the mean of three independent experiments. **, $\mathrm{P}<0.05$, ****, $\mathrm{P}<0.0001$ in intergroup comparisons.

(Figure 2B). These results indicate an inhibitory effect of hsa-miR-195-5p on TC cell proliferation.

\section{bsa-miR-195-5p over-expression induced cell cycle arrest at G0/G1 phase}

To deeply explore the mechanism underlying the antiproliferative effect of hsa-miR-195-5p on TC cell, flow cytometry for cell cycle analysis was performed. The results (Figure 3A) showed that over-expression of hsa-miR-195-5p significantly increased the frequency of TPC- 1 cells at the G0/G1 phase, which implied that hsa-miR-195-5p overexpression induced cell cycle arrest at the G0/G1 phase.

To confirm whether hsa-miR-195-5p could regulate the expression of cell cycle related markers at the molecular level, the levels of cyclin D1, a cell cycle related regulatory proteins and p21, a cell cycle inhibitor were simultaneously detected by western blotting. As expected, results indicated that over-expression of hsa-miR-195-5p markedly hindered the expression of cyclin D1 and up-regulated p21 while its inhibition triggered the opposite effects (Figure 3B). This observation supported the inhibitory effect of hsa-miR-195$5 \mathrm{p}$ on TC cell proliferation and suggested that hsa-miR- 195-5p may induce cell cycle arrest at the G0/G1 phase.

\section{Cyclin D1 is a direct target of bsa-miR-195-5p}

In order to understand the molecular mechanism on cell cycle and cell proliferation regulation by hsa-miR-195-5p, we used TargetScan 6.2 to predict target genes of hsa-miR$195-5 \mathrm{p}$. The results revealed that cyclin D1 was a potential target of hsa-miR-195-5p (Figure 4A). Subsequently, luciferase reporter assay was performed to examine whether there is a possible binding of hsa-miR-195-5p to 3'-UTR of cyclin D1 mRNA. As shown in Figure 4B, co-transfection of the reporter plasmid harboring the wild type 3'-UTR of cyclin D1 mRNA with hsa-miR-195-5p mimics resulted in a remarkable inhibition of luciferase activity contrary to the activity measured in cells co-transfected with cyclin D1 3 '-UTR-Mut and hsa-miR-195-5p mimics. These findings imply that hsa-miR-195-5p negatively regulates cyclin D1 by directly binding to its 3'-UTR in TC cells.

In addition, to check whether hsa-miR-195-5p exerts its function at the post-transcriptional level, cyclin D1 mRNA and protein levels were measured. The results showed that over-expression of hsa-miR-195-5p did not affect the cyclin 

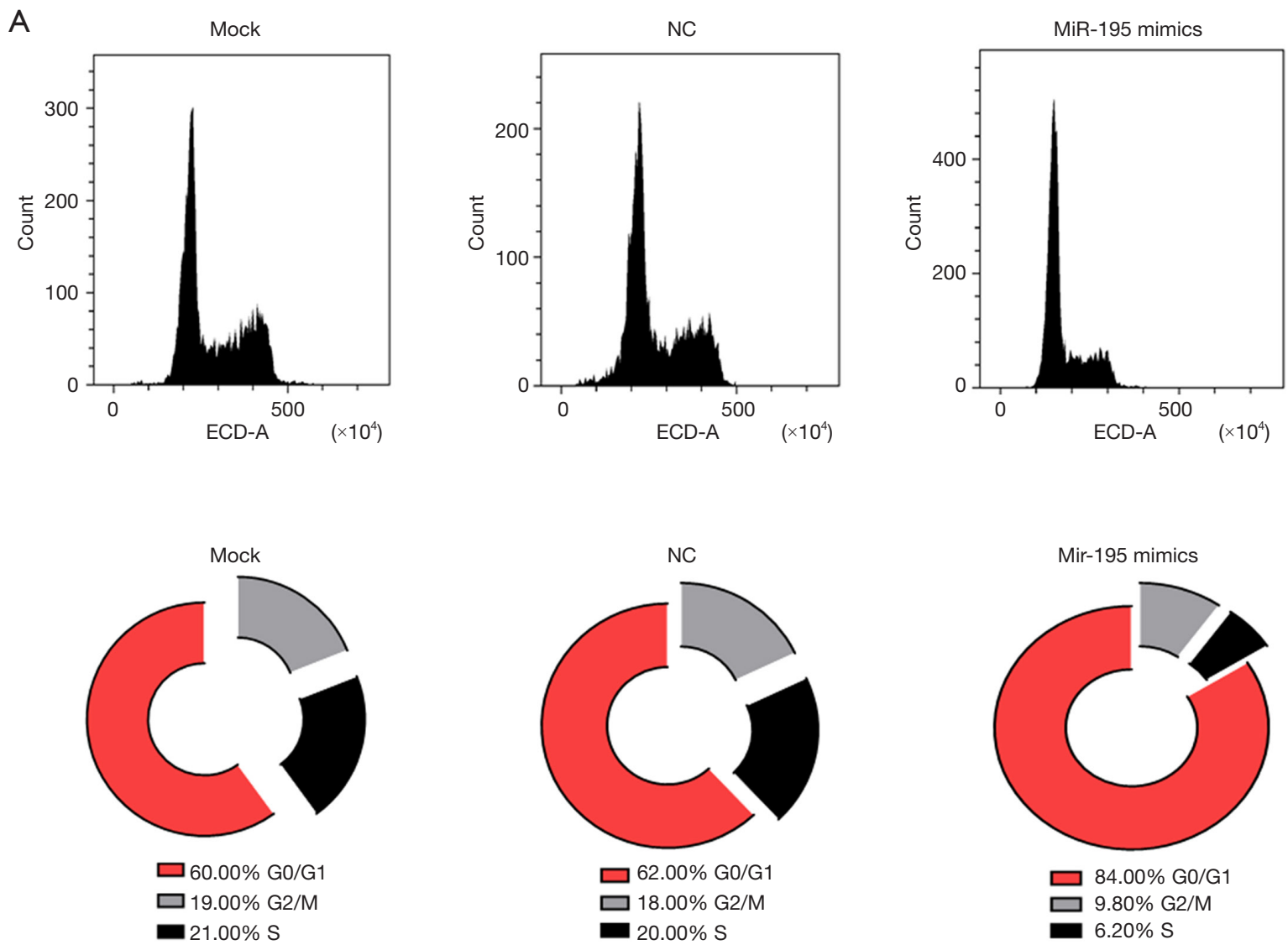

B
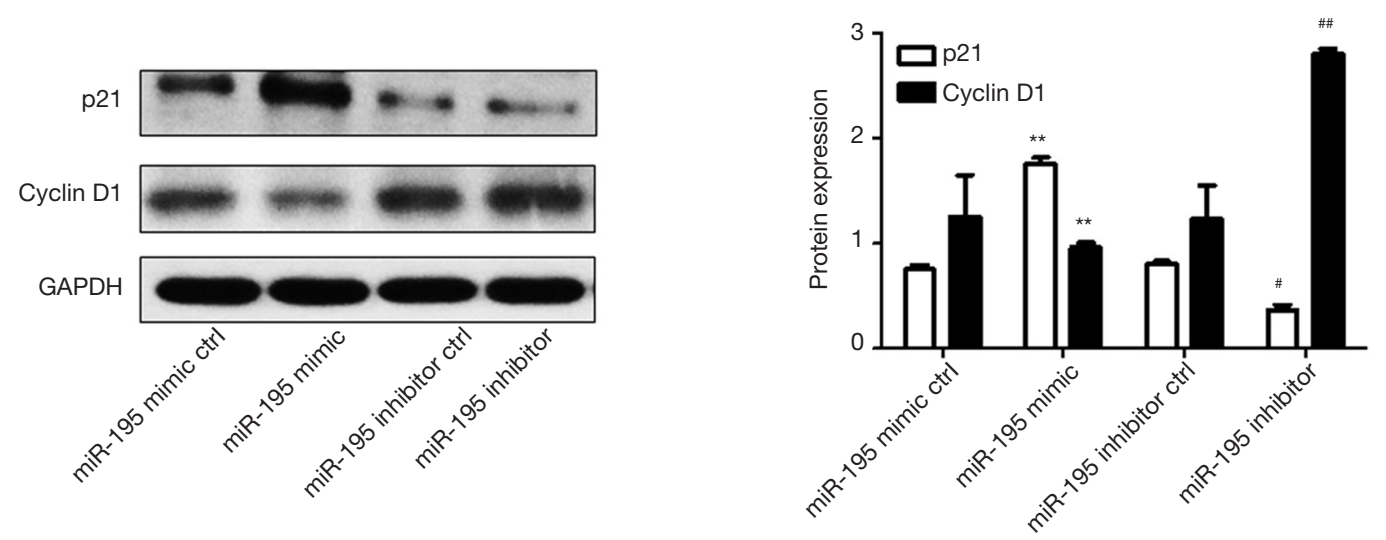

Figure 3 Hsa-miR-195-5p induces cell cycle arrest at G0/G1 phase by inhibition of cyclin D1 expression and upregulation of p21 expression in TPC-1 cells. (A) Flow cytometry analysis of cell cycle and quantitative representation. (B) Effects of hsa-miR-195-5p mimics on the endogenous cyclin D1 and p21 protein levels. TPC-1 cells were co-transfected with hsa-miR-195-5p mimics or negative control oligonucleotides. The expression of cyclin D1 and p21 was in cells was evaluated by Western blotting. GAPDH was used as loading control. The data were subjected to two-way ANOVA analysis. ${ }^{* *}, \mathrm{P}<0.01$ compared with mimic control, ${ }^{\#}, \mathrm{P}<0.05,{ }^{\# \#}, \mathrm{P}<0.01$ compared with inhibitor control; NC, negative control oligonucleotides. 
A

\author{
Position 1961-1967 of CCND1 3' UTR \\ hsa-miR-195-5p \\ Position 1961-1967 of CCND1 3' UTR -Mut
}

B

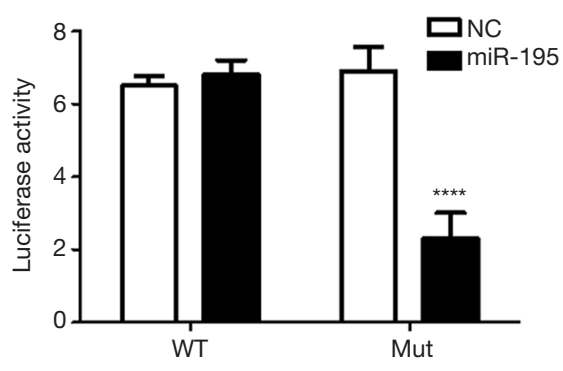

$\mathrm{D}$



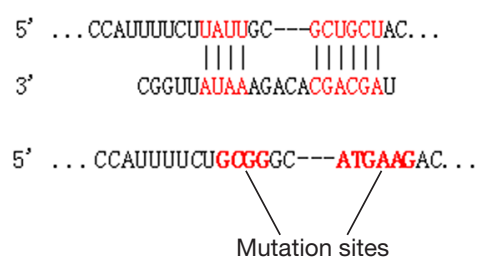

C



E

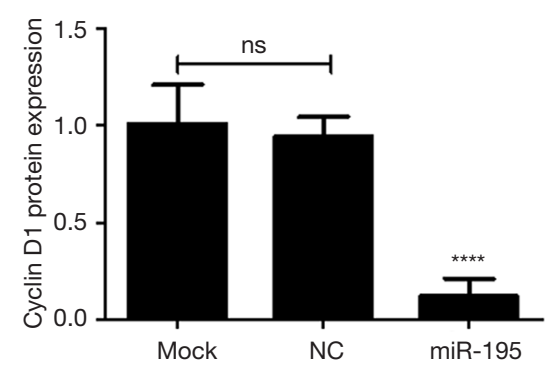

Figure 4 hsa-miR-195-5p suppresses cyclin D1 expression by directly targeting the cyclin D1 3 '-UTR in TPC-1 cells. (A) TargetScan prediction of hsa-miR-195-5p targeting of cyclin D1 and Mutagenesis of cyclin D1 3'-UTR. (B) Luciferase reporter assay. (C) Effects of hsamiR-195-5p mimics on the endogenous cyclin D1 mRNA levels. TPC-1 cells were cotransfected with hsa-miR-195-5p mimics or negative control oligonucleotides. Forty-eight hours after transfection, cells were isolated, the expression of cyclin D1 was analyzed by qRT-PCR. $(\mathrm{D}, \mathrm{E})$ Effects of hsa-miR-195-5p on the endogenous cyclin D1 protein levels. Forty-eight hours after transfection with the hsa-miR-195-5p mimics or negative control oligonucleotides, proteins were isolated from TPC-1 cells and analyzed by Western blotting, with GAPDH as an internal control. ${ }^{* * * *}, \mathrm{P}<0.0001$ compared with respective controls. NC, negative control oligonucleotides.

D1 mRNA levels (Figure 4C). Nevertheless, the protein level of cyclin D1 was significantly decreased in cells overexpressing hsa-miR-195-5p (Figure 4D,E). We inferred that hsa-miR-195-5p regulates cyclin D1 expression posttranscriptionally.

\section{Silencing of cyclin D1 reversed the inbibitory effects of bsa-miR-195-5p on TC cell proliferation}

In order to investigate the role of cyclin D1 in the TPC-
1 cells over-expressing hsa-miR-195-5p, we transfected the TPC-1 cells harboring hsa-miR-195-5p mimics with cyclin D1 siRNA to silence the expression of cyclin D1. Western blot analysis confirmed the efficiency of silencing (Figure 5A). In MTT assay, we found that silencing of cyclin D1 in hsa-miR-195-5p mimics-transfected TPC-1 cells reversed the anti-proliferative effect of hsa-miR-195-5p (Figure 5B). Further analysis confirmed that after silencing of cyclin D1, the cell cycle arrest caused by hsa-miR-195$5 \mathrm{p}$ was equally reversed (Figure 5C). These data support the 
A



B

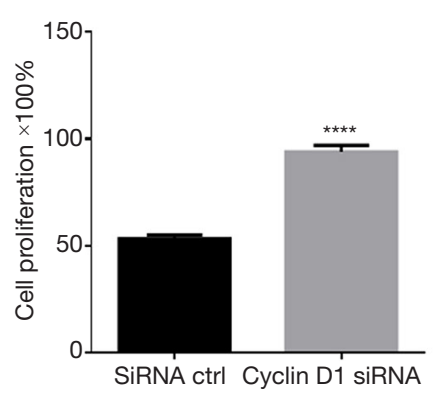

C
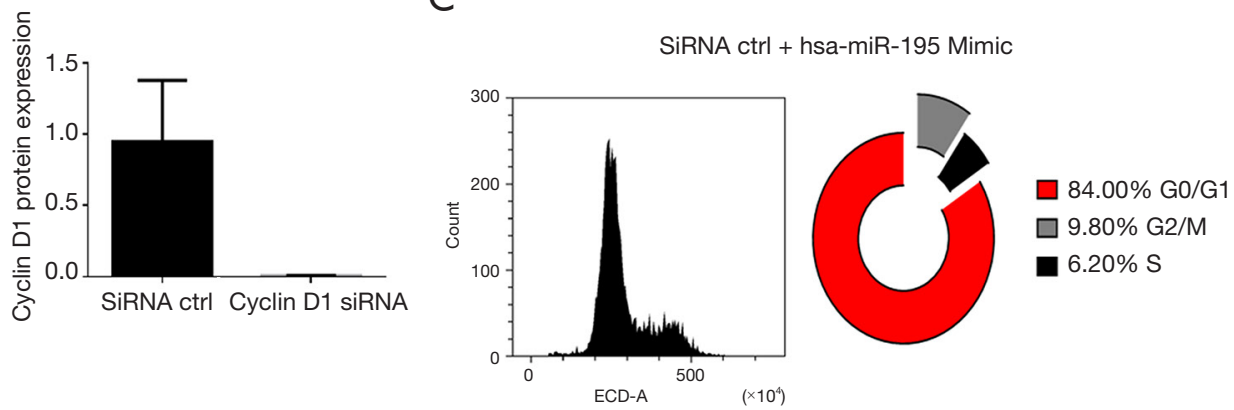

Cyclin D1 siRNA + hsa-miR-195 Mimic

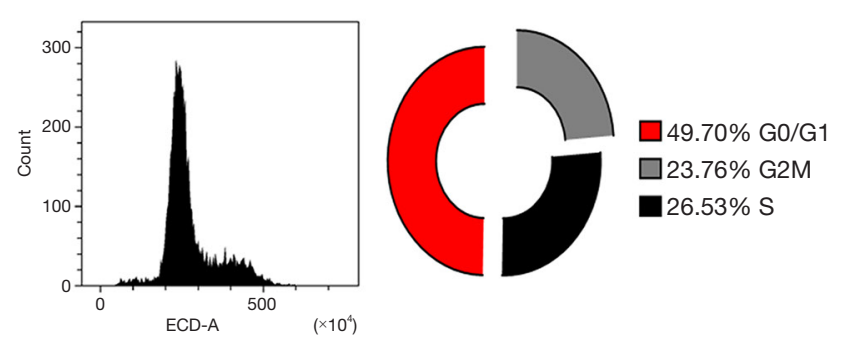

Figure 5 Cyclin D1 up-regulation is required for hsa-miR-195-5p inhibitor induced proliferation of TC cells. (A) Western blot analysis verified that silencing cyclin D1 effectively inhibited the expression of cyclin D1 TPC-1 cells. (B) Increased cell proliferation in hsa-miR195-5p mimics-transfected TPC-1 cells. (C) Hsa-miR-195-5p induced cell cycle arrest is mediated through down-regulation of cyclin D1.

idea that hsa-miR-195-5p inhibited TC cell proliferation by prompting cell cycle arrest at the G0/G1 phase through interference of cyclin D1 expression.

\section{Discussion}

MicroRNAs (miRNAs) are a group of novel gene regulatory factors, which play an important role in the process of tumor cells growth. Clinical evidence has shown that miRNAs are abnormally expressed in a variety of human tumors (35).

Latest studies indicated that miR-195 expression is considerably decreased in breast cancer and miR-195 acts as a tumor suppressor (36). It was also reported that the expression of miR-195 in adrenocortical carcinoma is significantly down-regulated, and the down-regulated level is positively correlated with poor prognosis (37). Comparable reports linked the down-regulation of miR195 in colorectal cancer with lymph node metastasis (38). Moreover, studies have demonstrated the involvement of miR-195 in acquired temozolomide resistance in glioblastoma multiform cells (39). Nonetheless, the significance of hsa-miR-195-5p in TC pathogenesis and its plausible application as a therapeutic target for this disorder has yet to be assessed.

In the present study, we measured the expression level of hsa-miR-195-5p in TC cell lines and evaluated its regulatory role on cell proliferation. Our findings showed that hsa-miR-195-5p expression was markedly decreased in TC cell lines compared with normal cells. This result also confirms the previous research results (36-39). Moreover, we discovered that over-expression of hsa-miR-195-5p markedly blocked TC cell proliferation by promoting cell cycle arrest at the G0/G1 phase, which was further confirmed by its regulatory effects on cyclin D1 and p21 protein. This result is consistent with that of Furuta et al. (40) who demonstrated that the molecular pathways regulating cell cycle progression are characteristically altered by decreased expression of miR-195, thus instigating abnormal cell proliferation in hepatocarcinogenesis.

In further experiments, we found that hsa-miR-195-5p inhibited TC cell proliferation via negative regulation of cyclin D1 in post-translational level. We also found that hsa-miR-195-5p over-expression was accompanied with increased expression of $\mathrm{p} 21$. Therefore, we speculated that hsa-miR-195-5p might inhibit TC cell proliferation by up-regulating p21 and down-regulating cyclin D1. The down-regulation of cyclin D1 was probably mediated by 
direct binding of hsa-miR-195-5p to its 3'-UTR or by the up-regulated p21 caused by hsa-miR-195-5p through an unknown mechanism. Accordingly, we found that hsamiR-195-5p might regulate TC cell proliferation via modulation of the p21/cyclin D1-pathway. The present observation was consistent with the views of Luo et al. (41) indicating the implications of cyclin D1/p21 pathway in the induction of cell cycle arrest and subsequent inhibition of cell proliferation. The current findings were also similar to those of former works considering that cyclins was targets for miR-195, which trigger cell cycle arrest at the G1/S phase (42-44).

Cyclin D1, a critical protein involved in cell cycle regulation, is up-regulated in most human tumors including TC $(45,46)$. It has also been reported that cyclin D1 plays a key role in the formation of activated CDK4/CDK6 complex, which in turn, initiates phosphorylation of $\mathrm{pRb}$ $(47,48)$. Consistent with these reports, our data show that cyclin D1 was down-regulated in TPC-1 cells overexpressing hsa-miR-195-5p mimic. Furthermore, silencing of cyclin D1 in TPC-1 cells transfected with hsa-miR-195$5 \mathrm{p}$ mimics reversed the negative effect of hsa-miR-195$5 \mathrm{p}$ on cell proliferation, which implies that hsa-miR-195$5 \mathrm{p}$ inhibited TC cell proliferation, partly at least, by direct targeting of cyclin D1.

\section{Conclusions}

Conclusively, our present study indicated that hsa-miR$195-5 \mathrm{p}$ is a candidate tumor-suppressor miRNA in TC and that the hsa-miR-195-5p/p21/cyclin D1 pathway could be a potential therapeutic target for TC.

\section{Acknowledgments}

Funding: This study was supported by Fujian medical innovation project of health department (2016-ZQN-54); Quanzhou High-level Personnel Foundation (2017Z005); Quanzhou Natural Foundation (2016Z052).

\section{Footnote}

Data Sharing Statement: Available at http://dx.doi. org/10.21037/tcr-20-1083

Conflicts of Interest: All authors have completed the ICMJE uniform disclosure form (available at http://dx.doi. org/10.21037/tcr-20-1083). DL reports grants from
Quanzhou Natural Foundation, during the conduct of the study. XW reports grants from Fujian medical innovation project of health department, grants from Quanzhou Highlevel Personnel Foundation, during the conduct of the study. The other authors have no conflicts of interest to declare.

Ethical Statement: The authors are accountable for all aspects of the work in ensuring that questions related to the accuracy or integrity of any part of the work are appropriately investigated and resolved.

Open Access Statement: This is an Open Access article distributed in accordance with the Creative Commons Attribution-NonCommercial-NoDerivs 4.0 International License (CC BY-NC-ND 4.0), which permits the noncommercial replication and distribution of the article with the strict proviso that no changes or edits are made and the original work is properly cited (including links to both the formal publication through the relevant DOI and the license). See: https://creativecommons.org/licenses/by-nc-nd/4.0/.

\section{References}

1. Pacini F, Schlumberger M, Dralle H, et al. European consensus for the management of patients with differentiated thyroid carcinoma of the follicular epithelium. Eur J Endocrinol 2006;154:787-803.

2. Teng $W$, Shan Z, Teng X, et al. Effect of iodine intake on thyroid diseases in china. $\mathrm{N}$ Engl J Med 2006;354:2783-93.

3. Leenhardt L, Grosclaude P, Chérié-Challine L. Increased incidence of thyroid carcinoma in france: A true epidemic or thyroid nodule management effects? Report from the french thyroid cancer committee. Thyroid 2004;14:1056-60.

4. Chen AY, Jemal A, Ward EM. Increasing incidence of differentiated thyroid cancer in the united states, 19882005. Cancer 2009;115:3801-7.

5. Drozd VM, Saenko VA, Brenner AV, et al. Major factors affecting incidence of childhood thyroid cancer in belarus after the chernobyl accident: Do nitrates in drinking water play a role? PLoS One 2015;10:e0137226.

6. Harris PJ, Bible KC: Emerging therapeutics for advanced thyroid malignancies: Rationale and targeted approaches. Expert Opin Investig Drugs 2011;20:1357-75.

7. Hu Y, Wang H, Chen E, et al. Candidate micrornas as biomarkers of thyroid carcinoma: A systematic review, 
meta-analysis, and experimental validation. Cancer Med 2016;5:2602-14.

8. Ab Mutalib NS, Othman SN, Mohamad Yusof A, et al. Integrated microrna, gene expression and transcription factors signature in papillary thyroid cancer with lymph node metastasis. PeerJ 2016;4:e2119.

9. Dong S, Meng X, Xue S, et al. Microrna-141 inhibits thyroid cancer cell growth and metastasis by targeting insulin receptor substrate 2. Am J Transl Res 2016;8:1471-81.

10. Pohl M, Grabellus F, Worm K, et al. Intermediate microrna expression profile in graves' disease falls between that of normal thyroid tissue and papillary thyroid carcinoma. J Clin Pathol 2017;70:33-9.

11. Qian Y, Wang X, Lv Z, et al. Microrna126 is downregulated in thyroid cancer cells, and regulates proliferation, migration and invasion by targeting cxcr4. Mol Med Rep 2016;14:453-9.

12. Qiu W, Yang Z, Fan Y, et al. Microrna-613 inhibits cell growth, migration and invasion of papillary thyroid carcinoma by regulating sphk2. Oncotarget 2016;7:39907-15.

13. Samsonov R, Burdakov V, Shtam T, et al. Plasma exosomal mir-21 and mir-181a differentiates follicular from papillary thyroid cancer. Tumour Biol 2016;37:12011-21.

14. Yi L, Yuan Y. Microrna-618 modulates cell growth via targeting pi3k/akt pathway in human thyroid carcinomas. Indian J Cancer 2015;52 Suppl 3:E186-9.

15. Yue K, Wang X, Wu Y, et al. Microrna-7 regulates cell growth, migration and invasion via direct targeting of pak1 in thyroid cancer. Mol Med Rep 2016;14:2127-34.

16. Schmitz KJ, Helwig J, Bertram S, et al. Differential expression of microrna-675, microrna-139-3p and microrna-335 in benign and malignant adrenocortical tumours. J Clin Pathol 2011;64:529-35.

17. Li L, Piontek K, Ishida M, et al. Extracellular vesicles carry miR-195 to intrahepatic cholangiocarcinoma and improve survival in a rat model. Hepatology 2017;65:501-514.

18. Liu C, Guan H, Wang Y, et al. MiR-195 inhibits emt by targeting fgf 2 in prostate cancer cells. PLoS One 2015;10:e0144073.

19. Luo Q, Zhang Z, Dai Z, et al. Tumor-suppressive microrna-195-5p regulates cell growth and inhibits cell cycle by targeting cyclin dependent kinase 8 in colon cancer. Am J Transl Res 2016;8:2088-96.

20. Shen YH, Xie ZB, Yue AM, et al. Expression level of microrna-195 in the serum of patients with gastric cancer and its relationship with the clinicopathological staging of the cancer. Eur Rev Med Pharmacol Sci 2016;20:1283-7.

21. Wang M, Zhang J, Tong L, et al. MiR-195 is a key negative regulator of hepatocellular carcinoma metastasis by targeting fgf 2 and vegfa. Int J Clin Exp Pathol 2015;8:14110-20.

22. Wang Y, Zhang X, Zou C, et al. MiR-195 inhibits tumor growth and angiogenesis through modulating irs1 in breast cancer. Biomed Pharmacother 2016;80:95-101.

23. Ye S, Song $W, X u X$, et al. Igf2bp2 promotes colorectal cancer cell proliferation and survival through interfering with raf-1 degradation by miR-195. FEBS Lett 2016;590:1641-50.

24. Zhang X, Tao T, Liu C, et al. Downregulation of miR-195 promotes prostate cancer progression by targeting hmgal. Oncol Rep 2016;36:376-82.

25. Zhao N, Li S, Wang R, et al. Expression of microrna-195 is transactivated by sp1 but inhibited by histone deacetylase 3 in hepatocellular carcinoma cells. Biochim Biophys Acta 2016;1859:933-42.

26. Zhou Q, Han LR, Zhou YX, et al. MiR-195 suppresses cervical cancer migration and invasion through targeting smad3. Int J Gynecol Cancer 2016;26:817-24.

27. Jin J, Wang C, Ouyang Y, et al. Elevated miR-195$5 \mathrm{p}$ expression in deep vein thrombosis and mechanism of action in the regulation of vascular endothelial cell physiology. Exp Ther Med 2019;18:4617-24.

28. Li P, Zhai P, Ye Z, et al. Differential expression of miR$195-5 \mathrm{p}$ in collapse of steroid-induced osteonecrosis of the femoral head. Oncotarget 2017;8:42638-47.

29. Zheng J, Xu T, Chen F, et al. MiRNA-195-5p Functions as a Tumor Suppressor and a Predictive of Poor Prognosis in Non-small Cell Lung Cancer by Directly Targeting CIAPIN1. Pathol Oncol Res 2019;25:1181-90.

30. Pan SS, Zhou HE, Yu HY, et al. MiR-195-5p inhibits the cell migration and invasion of cervical carcinoma through suppressing ARL2. Eur Rev Med Pharmacol Sci 2019;23:10664-71.

31. Wang F, Jiang C, Sun Q, et al. MiR-195 is a key regulator of raf1 in thyroid cancer. Onco Targets Ther 2015;8:3021-8.

32. Maroof H, Irani S, Arianna A, et al. Interactions of Vascular Endothelial Growth Factor and p53 with miR195 in Thyroid Carcinoma: Possible Therapeutic Targets in Aggressive Thyroid Cancers. Curr Cancer Drug Targets 2019;19:561-70.

33. Yin Y, Hong S, Yu S, et al. MiR-195 Inhibits Tumor Growth and Metastasis in Papillary Thyroid Carcinoma 
Cell Lines by Targeting CCND1 and FGF2. Int J

Endocrinol 2017;2017:6180425.

34. Du P, Liu F, Liu Y, et al. Linc00210 enhances the malignancy of thyroid cancer cells by modulating miR195-5p/IGF1R/Akt axis. J Cell Physiol 2020;235:1001-12.

35. Jin S, Dai Y, Li C, et al. Microrna-544 inhibits glioma proliferation, invasion and migration but induces cell apoptosis by targeting park7. Am J Transl Res 2016;8:1826.

36. Li D, Zhao Y, Liu C, et al. Analysis of miR-195 and mir497 expression, regulation and role in breast cancer. Clin Cancer Res 2011;17:1722-30.

37. Soon PSH, Tacon LJ, Gill AJ, et al. MiR-195 and mir$483-5 \mathrm{p}$ identified as predictors of poor prognosis in adrenocortical cancer. Clin Cancer Res 2009;15:7684-92.

38. Wang X, Wang J, Ma H, et al. Downregulation of miR-195 correlates with lymph node metastasis and poor prognosis in colorectal cancer. Med Oncol 2012;29:919-27.

39. Ujifuku K, Mitsutake N, Takakura S, et al. MiR-195, mir-455-3p and mir-10a* are implicated in acquired temozolomide resistance in glioblastoma multiforme cells. Cancer Lett 2010;296:241-8.

40. Furuta M, Kozaki KI, Tanimoto K, et al. The tumorsuppressive mir-497-195 cluster targets multiple cellcycle regulators in hepatocellular carcinoma. PloS One 2013;8:e60155.

41. Luo J, Zhang C, Wang C, et al. Miz-1 promotes the

Cite this article as: Liu D, Li P, Wang X, Wang W. hsa-miR195-5p inhibits cell proliferation of human thyroid carcinoma cells via modulation of p21/cyclin D1 axis. Transl Cancer Res 2020;9(9):5190-5199. doi: 10.21037/tcr-20-1083 proliferation of esophageal cancer cells via suppression of p21 and release of p21-arrested cyclin d1. Oncol Rep 2016;35:3532-40.

42. Hui W, Yuntao L, Lun L, et al. Microrna-195 inhibits the proliferation of human glioma cells by directly targeting cyclin d1 and cyclin e1. PLoS One 2013;8:e54932.

43. Sekiya Y, Ogawa T, Iizuka M, et al. Down-regulation of cyclin e1 expression by microrna-195 accounts for interferon- $\beta$-induced inhibition of hepatic stellate cell proliferation. J Cell Physiol 2011;226:2535-42.

44. He JF, Luo YM, Wan XH, et al. Biogenesis of mirna-195 and its role in biogenesis, the cell cycle, and apoptosis. J Biochem Mol Toxicol 2011;25:404-8.

45. Marampon F, Gravina G, Ju X, et al. Cyclin d1 silencing suppresses tumorigenicity, impairs DNA double strand break repair and thus radiosensitizes androgenindependent prostate cancer cells to DNA damage. Oncotarget 2016;7:5383-400.

46. Burandt E, Grünert M, Lebeau A, et al. Cyclin d1 gene amplification is highly homogeneous in breast cancer. Breast Cancer 2016;23:111-9.

47. Bartkova J, Lukas J, Müller H, et al. Cyclin d1 protein expression and function in human breast cancer. Int J Cancer 1994;57:353-61.

48. Finn RS, Aleshin A, Slamon DJ. Targeting the cyclindependent kinases (cdk) 4/6 in estrogen receptor-positive breast cancers. Breast Cancer Res 2016;18:17. 